\title{
Low-profile Flexible Perovskite based Millimetre Wave Antenna
}

\author{
Abdoalbaset Abohmra ${ }^{1}$, Fizzah Jilani ${ }^{2}$, Hasan Abbas ${ }^{3}$, Rami Ghannam ${ }^{1}$,Hadi Heidari ${ }^{1}$, Muhammad Ali Imran ${ }^{1}$ and Qammer H. Abbasi ${ }^{1}$ \\ ${ }^{1}$ School of Electrical and Electronic Engineering, Glasgow University, Glasgow, G12 8QQ, UK \\ ${ }^{2}$ School of Electronic Engineering and Computer Science Queen Mary University of London, London E1 4NS, UK \\ ${ }^{3}$ Department of Physics, Texas A \& M University at Qatar, Doha, Qatar \\ 1'Email: 2356006@ student.ac.uk, ${ }^{2}$ Email: s.f.jillani@qmul.ac.uk, ${ }^{3}$ Email: hasan.abbas@qatar.tamu.edu, \\ ${ }^{1}$ Email: $\{$ Hadi.Heidari, Rami.Ghannam, Muhammad.Imran, Qammer.Abbasi\}@glasgow.ac.uk
}

\begin{abstract}
This paper presents the potential of perovskite antenna in the millimeter band. New materials have been developed to obtain suitable chemical characteristics to offer several advantages including simple structure, high operating bandwidth, high gain, compact and low-profile antennas in the millimeter range. The performance of antennas fabricated using these materials is analyzed here. Simulated results of the antenna prototype show a wide bandwidth of 26-33 GHz. Moreover, simulation evaluation of peak gain of the antenna is $1.443 \mathrm{~dB}$ at 28.8 GHz. Low efficiency of $45 \%$ in the range of operation presents as the main drawback of this design. The analysis is further extended to examine the impact of different flexible substrate materials. The thickness of the patch is also examined to optimize the antenna radiation performance.
\end{abstract}

\section{INTRODUCTION}

The millimeter waves (MMW) spectrum constitutes the frequency range from around $20 \mathrm{GHz}$ to $100 \mathrm{GHz}$ and is regarded as the potential candidate for future $5 \mathrm{G}$ networks [1]. The MMW bands propose a fast and seamless communication and offer data rate up to $1 \mathrm{Gbps}$ to be used for a wide range of wireless services particularly in telecommunication systems. The MMW range has been used for military communication and radar application for years [2]. The increase in the gain and efficiency of an antenna are counterpoise by higher propagation loss at high frequencies. Flexible substrates offer the advantages of lighter weight, thinner structures, low fabrication cost, and conformity to be a part of wearables, body-centric as well as medical applications [3].

Perovskites have been widely investigated all around the world given their fascinating properties however their application related to photovoltaic applications and solar. The unique properties of perovskite have allowed the development of numerous novel applications, concepts, and devices. Perovskite materials display many fascinating characteristics in terms of superconductivity, ferroelectricity and high thermopower [4]. The chemical formula of Perovskite $\left(\mathrm{CH}_{3} \mathrm{NH}_{3} \mathrm{PbI}_{3}\right)$ suggests that it belongs to the family of methylammonium-trihalogeno-plumbates, discovered by Weber in 1978 [5]. Recently organic/inorganic lead and tin halide perovskites have been identified as promising materials, due to their large absorption coefficient, high charge carrier mobility, and diffusion length indicating that this material could be a potential candidate for the antenna design.
Controlling the physical properties in perovskite is quite challenging in the design of next generation functional devices meant for high frequencies [6]. Various environmental factors including moisture, ultraviolet light, and thermal stress, play a key role in the instability of perovskite materials [7].

In this paper, we investigated MMW antenna design based on perovskite patch integrated on a flexible substrate. Moreover, the fundamental electromagnetic properties of perovskite material are evaluated by using perovskite film as a radiating element in the antenna. In addition, different flexible substrate materials are used to optimize the antenna performance. It has been observed that the designed antenna constitutes the advantages of wide bandwidth and conformity as well as contributes in the development of antennas based on advanced materials.

\section{PEROVSKITE ANTENNA DESIGN}

The proposed antenna was designed using CST microwave studio (2018). The performance of the designed perovskitebased antenna geometry is examined at $293 \mathrm{~K}$, which is the room temperature. The antenna geometry consists of a perovskite patch, ground plane, and a flexible dielectric substrate film. Perovskite material is created in the CST studios by deploying the values of the measured complex permittivity demonstrated in [7]. To provide impedance matching, the $50 \Omega$-matched microstrip feed is positioned at the centre, as the inset-feed topology provided with a wave port shown in Fig 1. The ground plane and feedline are made of copper, while Rogers 3006 (dielectric constant, $\varepsilon_{\mathrm{r}}=6.5$ and loss tangent, $\tan \delta=0.0025$ ) is used as the flexible substrate, the dimensions of the substrate are $70 \times 70 \times 1.57 \mathrm{~mm}^{3}$.

Fig. 2 shows the reflection coefficient $\left(S_{11}\right)$ profile of the designed antenna. $S_{11}$ dip has the minimum value $(-42 \mathrm{~dB})$ at $28 \mathrm{GHz}$. A high operating bandwidth in the range of 26-33 $\mathrm{GHz}$ has been achieved from the designed antenna by taking the $S_{11}$ of $-10 \mathrm{~dB}$ as a reference. The antenna design has also been investigated for different film thicknesses to examine the performance variation. The results of Fig. 3 show that the thickness of the thin-film perovskite surface has an impact on the antenna performance as the $S_{11}$ profile varies with the change in thickness. However, it is observed that the overall 
bandwidth remains conserved. The proposed antenna resonant at $2 \mathrm{G} \mathrm{GHz}$ as well as $28 \mathrm{GHz}$ bands. These two bands are significantly highlighted at different communication forum for the potential deployment of $5^{\text {th }}$ generation $(5 \mathrm{G})$ wireless networks. It has been observed that significant improvements in the magnitude of $S_{11}$ have been observed by increasing the film thickness of perovskite.

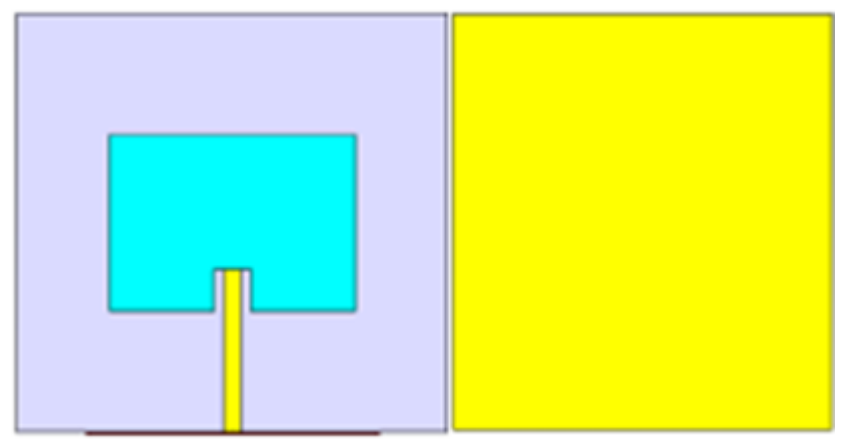

Figure 1. Perovskite based flexible MMW antenna design.

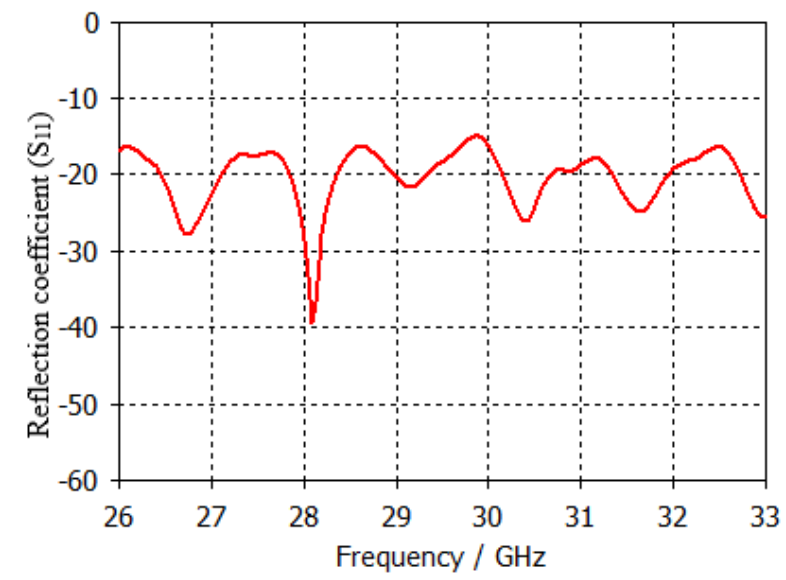

Figure 2. Reflection coefficient $\left(\mathrm{S}_{11}\right)$ simulation results of the purposed Perovskite based antenna.

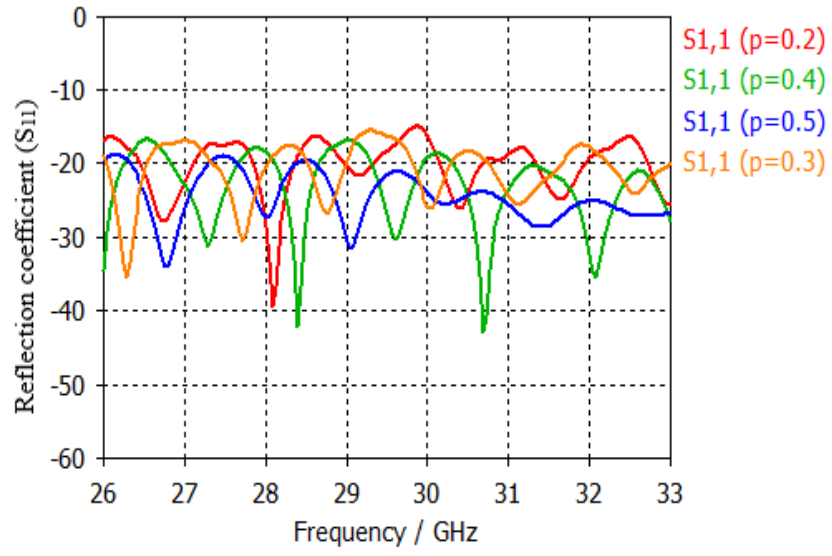

Figure 3. Reflection coefficient $\left(\mathrm{S}_{11}\right)$ simulation results of the proposed Perovskite based antenna for different patch thicknesses.
As the relative permittivity changed due to different substrate materials, $S_{11}$ is also changed as a result. In Fig. 4, three substrate materials are tested namely, paper $\left(\varepsilon_{\mathrm{r}}=2.31\right.$, orange curve), polyamide $\left(\varepsilon_{\mathrm{r}}=3.5\right.$, red curve) and Rogers $3006\left(\varepsilon_{\mathrm{r}}=6.5\right.$, blue curve $)$. It has been observed that when the permittivity increased from 2.31 (paper) to 6.5 (Rogers 3006), the resonant frequency shifted from $30.8 \mathrm{GHz}$ to $28.04 \mathrm{GHz}$. These selected substrate materials offer a high flexibility and lightweight making superlative substrate materials for wearable applications. The analysis presented in the Fig. 4, depicts that all the selected substrate films work perfectly, and the antenna resonate over a complete bandwidth of the desired frequency band with a $S_{11}$ magnitude lower than $-10 \mathrm{~dB}$.

The realized gain plotted against the frequency for the proposed antenna is shown in Fig. 5. The plot shows that 1.4 $\mathrm{dB}$ of gain has been achieved in the simulation at $28 \mathrm{GHz}$. Fig. 6 shows the voltage standing wave ratio (VSWR) which is below 2 which determines that a relatively better impedance matching with a lesser power reflection. The plot shows corresponding VSWR value of 1.017 is achieved at resonating frequencies of $28.04 \mathrm{GHz}$.

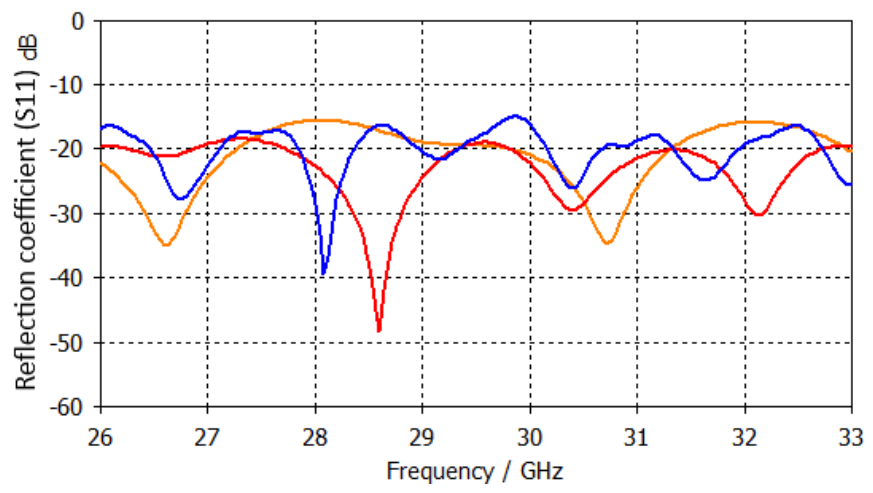

Figure 4. The effect of different permittivity on the Perovskite based antenna performance, Paper (orange curve), Polyamide (red curve), and Rogers 3006 (Blue curve).

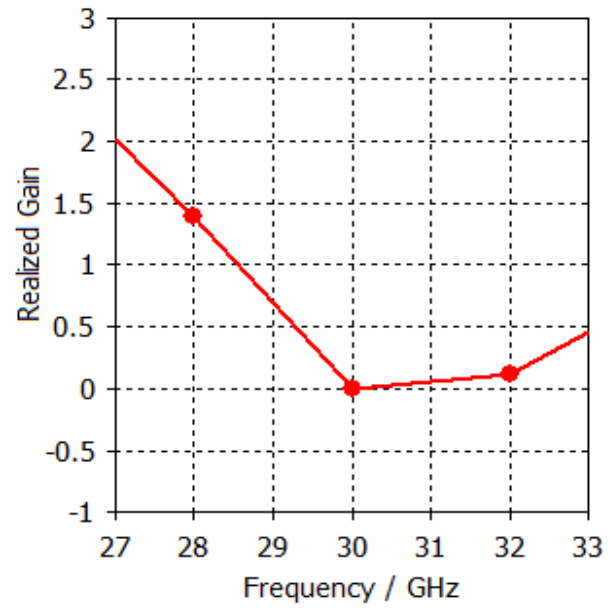

Figure 5. Simulated (in CST) realized gains against frequency of the Perovskite based antenna. 


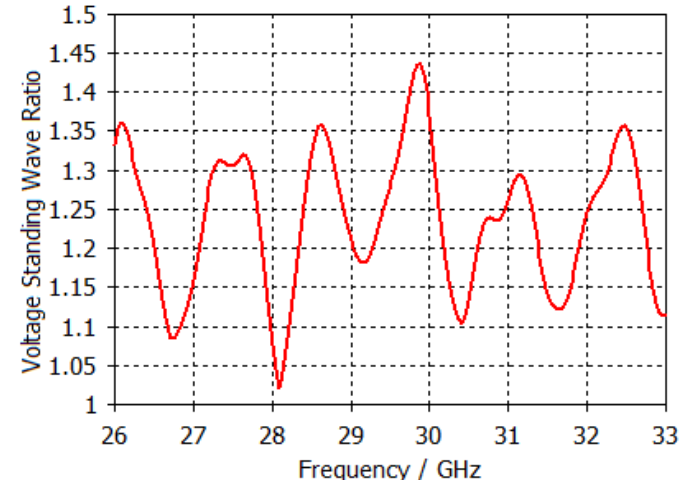

Figure 6. Voltage Standing Wave Ratio (VSWR) of the Perovskite based antenna.

Fig. 7 (a) and (b) show the simulated E-field and $\mathrm{H}$-field radiation patterns of the perovskite antenna at $28.04 \mathrm{GHz}$. Antenna efficiency is one of the most significant parameters at this high frequency. Based on the simulated results presented in Fig. 8, the antenna has a low efficiency of $45 \%$ at $28 \mathrm{GHz}$, which is much lesser as compared to the antenna based on copper metal. However, the efficiency could improve to 50\% and $60 \%$ by applying external bias voltage to increase the conductivity of this material. In addition, working at higher frequency would increase the conductivity of perovskite material and intrinsic motivation of charge carrier.

Farfield Directivity Abs $(\mathrm{Phi}=0)$

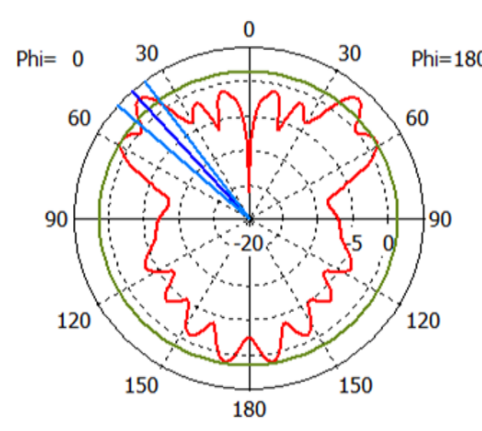

Frequency $=28.044 \mathrm{GHz}$ Main lobe magnitude $=3.44 \mathrm{~dB}$ Main lobe direction $=42.0 \mathrm{deg}$. Angular width $(3 \mathrm{~dB})=12.2 \mathrm{deg}$. Theta / Degree vs. dBi (a)

Farfield Directivity Abs (Phi=90)

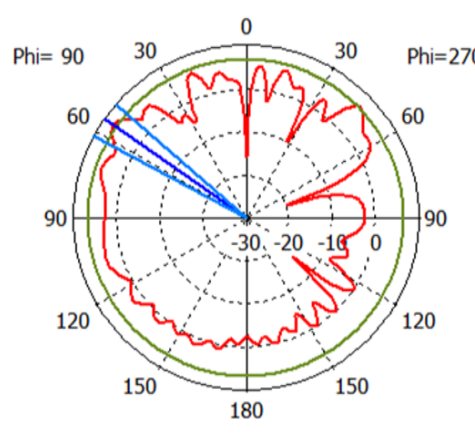

Theta / Degree vs. dBi

(b)

Figure 7. Simulated (in CST) radiation patterns of the proposed of the Perovskite (units: degrees and $\mathrm{dBi}$ ) where the red line is the electric field, the green line is the magnetic field and blue is angular width at $28.044 \mathrm{GHz}$.: (a) E-plane cut, (b) H-plane cut.

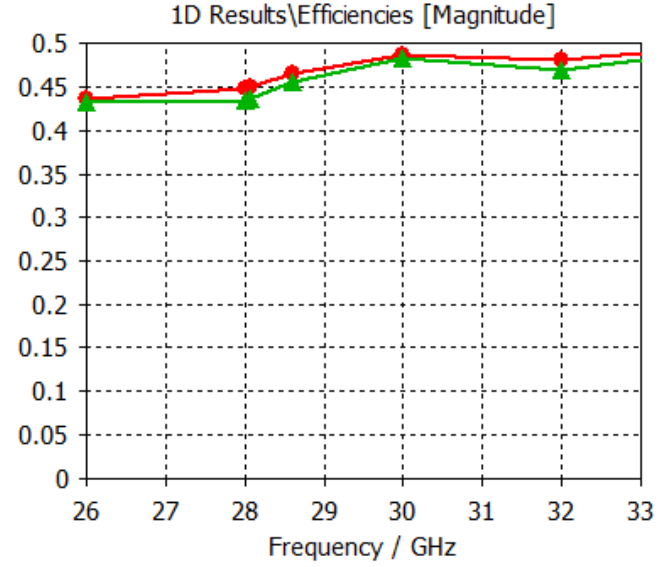

Figure 9. Simulated (in CST) efficiency of the proposed Perovskite based antenna.

\section{CONCLUSION AND FUTURE WORK}

In this paper, a fundamental proof of concept has been presented for the deployment of advanced materials such as perovskite for the antennas with a reasonable efficiency. The results show that the antenna offers a wide bandwidth of 26-33 $\mathrm{GHz}$, however the maximum efficiency achieved is $45 \%$. The results show that the proposed work has the potential for future studies on perovskite-based antenna to improve the efficiency further by means of doping or external biasing. These results pave the way for the use of perovskite-based antenna as an option to conventual lossy material in high frequency. The proposed antenna is compact, very easy to fabricate, and attractive for future wearable applications.

\section{ACKNOWLEDGMENT}

Authors are sincerely grateful to Libyan Government for this research support.

\section{REFERENCES}

[1] Q. Zhao and J. Li, "Rain attenuation in millimeter wave ranges," in IEEE Int. Symp. Antennas, Propag. EM Theory, 2006, pp. 1-4.

[2] H. Chu and Y. X. Guo, "A novel approach for millimeter-wave dielectric resonator antenna array designs by using the substrate integrated technology," IEEE Trans. Antennas Propag., vol. 65, no. 2, pp. 909-914, 2017.

[3] C. A. Balanis, 1992. Antenna theory: A review. Proceedings of the IEEE, vol. 80, no. 1, pp. 7-23.

[4] A. Kulkarni et al., "Mixed ionic electronic conducting perovskite anode for direct carbon fuel cells," Int. J. Hydrogen Energy, vol. 37, no. 24, pp. 19092-19102, 2012.

[5] N. K. Noel, et al., "Enhanced photoluminescence and solar cell performance via Lewis base passivation of organic-inorganic lead halide perovskites," ACS Nano, vol. 8, no.10, pp. 9815-9821, 2014.

[6] Z. Ahmad et al., "Instability in CH 3 NH 3 PBI 3 perovskite solar cells due to elemental migration and chemical composition changes," Sci. Rep., vol. 7, no. 1, pp.15406, 2017.

[7] M. A. Green, A. Ho-Baillie and H. J. Snaith, "The emergence of perovskite solar cells,” Nature Photonics, vol. 8, no. 7, pp. 506, 2014. 
